Revue

Revue de l'histoire des religions

de Ihistoire des religions

$4 \mid 2017$

Les discours sur les religions dans l'Empire romain : regards croisés entre " païens ", " juifs » et « chrétiens »

\title{
Le discours sur les religions chez Grégoire de Nazianze et Maxime le Confesseur, ou l'art de discréditer le « monothéisme » juif
}

Discourse on Religions in Gregory of Nazianzus and Maximus the Confessor, or the Art of Discrediting Jewish "monotheism"

\section{Christian Boudignon}

\section{OpenEdition}

\section{Journals}

Édition électronique

URL : http://journals.openedition.org/rhr/8834

DOI : $10.4000 /$ rhr.8834

ISSN : 2105-2573

Éditeur

Armand Colin

Édition imprimée

Date de publication : 1 décembre 2017

Pagination : 777-795

ISBN : 978-2-200-93128-5

ISSN : 0035-1423

Référence électronique

Christian Boudignon, « Le discours sur les religions chez Grégoire de Nazianze et Maxime le

Confesseur, ou l'art de discréditer le « monothéisme » juif », Revue de l'histoire des religions [En ligne], 4 | 2017, mis en ligne le 31 décembre 2019, consulté le 13 janvier 2021. URL : http:// journals.openedition.org/rhr/8834; DOI : https://doi.org/10.4000/rhr.8834 


\title{
Le discours sur les religions chez Grégoire de Nazianze et Maxime le Confesseur, ou l'art de discréditer le « monothéisme » juif
}

\begin{abstract}
Le discours sur les religions de Grégoire de Nazianze, d'abord ternaire (polythéisme, athéisme, judaïsme) devient binaire (judaïsme et hellénisme). Le judaïsme y occupe la catégorie qui correspond aujourd'hui au " monothéisme ». Une tentative originale de Grégoire consiste à recourir aux catégories politico-religieuses (polyarchie, monarchie, anarchie) et à introduire dans le "monothéisme " juif l'idée de rébellion de l'Un contre lui-même, ce qui aboutirait à un polythéisme. Refusant l'idée de dissension en Dieu, Maxime le Confesseur propose à partir des positions de Grégoire, un autre discours sur les religions où, face au polythéisme, le « monothéisme » juif serait réduit à l'athéisme, laissant ouverte pour le christianisme la catégorie du "monothéisme».
\end{abstract}

\section{Discourse on Religions in Gregory of Nazianzus and Maximus the Confessor, or the Art of Discrediting Jewish "monotheism"}

Gregory of Nazianzus's discourse on religions is at first ternary (polytheism, atheism, Judaism) and later becomes binary (Judaism and Hellenism). Judaism is not only a religion but also the central category for what we call "monotheism" today. But Gregory also makes an original attempt to skip this category in his political discourse on religions (polyarchy, monarchy, anarchy), and instead inserts into Jewish "monotheism" the Neoplatonist idea of the rebellion of the One against himself in order to dismiss it as a polytheism. Maximus the Confessor refuses the notion of dissent within God, and, based on Gregory's positions, proposes to reduce Jewish "monotheism" to atheism, so that Christianity may appear as the only real "monotheism". 
Dans son Dictionnaire philosophique, à l' article « Théologien »", Voltaire présente le portrait bien amusant d' « un vrai théologien qui possédait les langues de l'Orient». Après avoir passé sa vie à débrouiller les questions les plus confuses et notamment « comment l'Église chrétienne fut divisée depuis sa naissance en différents partis et comment la société dominante traita toutes les autres d'hérétiques », il devint véritablement savant, se défia de tout ce qu'il savait et «à sa mort avoua qu'il avait consumé inutilement sa vie». Sur les traces de ce théologien, qui est peut-être un autoportrait de Voltaire, passionné d'histoire du christianisme, je voudrais modestement réfléchir à la façon dont deux grandes figures du christianisme grec antique, Grégoire de Nazianze à la fin du $\mathrm{IV}^{\mathrm{e}}$ siècle et Maxime le Confesseur au début du VII ${ }^{\mathrm{e}}$ siècle, ont pensé la catégorie religieuse du «monothéisme », à défaut du mot qui ne fut inventé qu'en $1660^{2}$.

\section{GRÉGOIRE DE NAZIANZE ET SON DISCOURS SUR LES RELIGIONS}

\subsection{Discours ternaire : athéisme, judaïsme, polythéisme}

Grégoire de Nazianze ne s'intéresse pas à l'histoire des religions ou à la philosophie des religions en tant que telle. Il s'en sert seulement pour présenter sa position doctrinale comme une voie moyenne ${ }^{3}$, dans un de ses premiers discours, le Discours 2, prononcé à Nazianze en 362 :

1. Voltaire, Dictionnaire philosophique [1764], éd. Gerhardt Stenger, Paris, Flammarion, 2010, p. 503.

2. Pour une présentation générale de la question monothéiste, voir Christian Boudignon, " "Il est Dieu, unique" (Coran, 112) : comment penser le Dieu un ?», éd. Dionigi Albera, Katell Berthelot, Dieu, une enquête, Paris, Flammarion, 2013, p. 233-298.

3. Le thème du juste milieu a été étudié notamment par Jean Plagnieux, Saint Grégoire de Nazianze théologien, Paris, Cerf, 1951, p. 231 suiv. Salvatore Lilla, Dionigi l'Areopagita e il platonismo cristiano, Brescia, Morcelliana, 2005, p. 131, n. 371, rappelle que cette idée de « juste milieu » entre polythéisme grec et monarchisme judaïque se retrouve chez Grégoire de Nysse, Oratio catechetica magna, 3 (Patrologia Graeca 45, col. 17 D) et éd. Ekkehardus Mühlenberg,

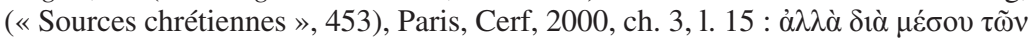


Il faut réellement marcher sur la voie royale $(\mathrm{Nb} 20,17)$ et faire attention à «ne dévier ni à droite ni à gauche » $(\operatorname{Pr} 4,27)$, comme le disent les Proverbes ${ }^{4}$.

Grégoire est tributaire de l'exégèse philonienne qui fait de la « voie royale » le juste milieu de la vie philosophique ${ }^{5}$. Grégoire compare explicitement la fonction du pasteur qui conduit spirituellement son troupeau à celui d'un équilibriste. Le discours sur les religions apparaît comme un discours pastoral, et en tant que tel, inévitablement politique, dans la mesure où le triomphe de la position orthodoxe, nicéenne, celle de Grégoire, est un enjeu de politique ecclésiastique qui courra jusqu'en 380 au moins.

Dans ce contexte, Grégoire hérite d'un discours sur les religions visiblement judéo-chrétien qui fait du judaïsme la voie médiane entre l'athéisme et le polythéisme. Le judaïsme apparaît donc comme le nom de ce que l'on appelle aujourd'hui le «monothéisme ». Ce discours sur les religions n'est pas remodelé pour définir le christianisme vis-à-vis des autres religions que sont le judaïsme et l'hellénisme / paganisme, comme l'a fait peu de temps auparavant un Eusèbe de Césarée ${ }^{6}$, mais pour classer les opinions doctrinales à l'intérieur du christianisme. C'est un point de vue extérieur : le christianisme n'a pas encore sa place dans le discours religieux. Le but est, en fait, de combattre l'opinion des « trop orthodoxes », les moines de Nazianze qui accusaient son père, déjà évêque de Nazianze, de compromission avec l'arianisme. Ainsi on lit un peu plus loin dans le même Discours 2 :

Comme en effet il y a trois maladies théologiques en ce moment, l'athéisme, le judaïsme et le polythéisme, dont Sabellius le Libyen a

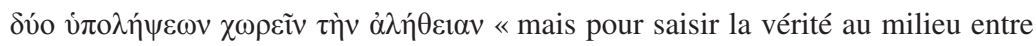
ces deux opinions ».

4. Grégoire de Nazianze, Discours, II, 34, éd. Jean Bernardi, (« Sources

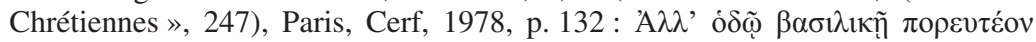

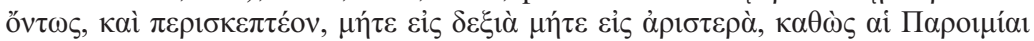

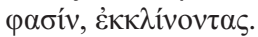

5. S. Lilla, Dionigil'Areopagita,p. 131,n. 371 rappelle que Philon d'Alexandrie avait appelé « voie royale », la voie qui conduisait à Dieu, voie identifiée à la vertu qui, comme l'enseignait Aristote, recherchait le juste milieu et refusait les excès opposés. S. Lilla renvoie aux traités de Philon : De posteritate Caini, 101 ; Quod Deus sit immutabilis, 159, 160 ; De migratione Abrahami, 146.

6. Voir l'intervention de Francesco Massa dans ce dossier et son article « Les théologies barbares chez Eusèbe de Césarée. Taxinomies et hiérarchies », Sagesses barbares. Échanges et réappropriation dans l'espace culturel gréco-romain, éd. Sydney H. Aufrère, Frédéric Möri, Genève, La Baconnière, 2016, p. 597-622. 
patronné la première, Arius l'Alexandrin la seconde, et de certaines gens trop orthodoxes de chez nous la troisième, quel sera mon discours ??

Le danger de cette présentation est qu'elle donne à ce qui sera la bête noire de Grégoire, l'arianisme, la position médiane que pourtant Grégoire voulait réclamer pour lui. Le christianisme nicéen n'est pas une catégorie dans ce discours sur les religions ; il apparaît seulement implicitement comme la seule opinion saine au milieu des « maladies théologiques », comme un dépassement des religions.

Cependant cette doctrine orthodoxe nicéenne reste suspendue dans le vide : c'est que Grégoire veut éviter le danger de faire de la position des « trop orthodoxes » une position médiane. Cela prouve qu'on est en pleine rhétorique : il n'y a pas de conflit doctrinal mais un simple conflit politique entre les moines de Nazianze et la famille de Grégoire sur l'attitude à avoir vis-à-vis de l'arianisme. Cette attitude rhétorique ne pouvait déboucher sur une position doctrinale solide. Elle sacrifie aux circonstances ce qui sera plus tard le souci de Grégoire, à savoir de faire de l'orthodoxie nicéenne la position médiane.

\subsection{Discours binaire : hellénisme et judaïsme}

À Constantinople en 380, Grégoire revient à un discours binaire sur les religions, paganisme / hellénisme et judaïsme dans la grande tradition paulinienne ${ }^{8}$. Ainsi dans le Discours 25, la description change légèrement :

[N'enseigne] ni trois principes, afin que ce ne soit pas le polythéisme païen/hellène, ni un seul principe mais juif : étroit pour ainsi dire, envieux et impuissant, c'est-à-dire le fait de ruiner la divinité en ellemême (idée qui a plu à ceux qui font sortir le Fils du Père mais le dissolvent ensuite en lui), ou bien le fait d'abolir les natures et de les bannir de la divinité (ce qui plaît aux sages d'aujourd'hui), comme si la divinité craignait la rébellion des natures et qu'elle ne fût pas douée de davantage de pouvoir que les créatures ${ }^{9}$.

7. Grégoire de Nazianze, Discours, II, 37, éd. J. Bernardi, p. 136-138 : Tpı̃̃v

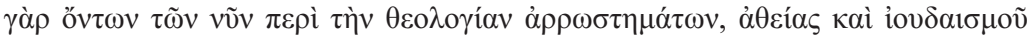

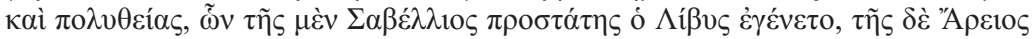

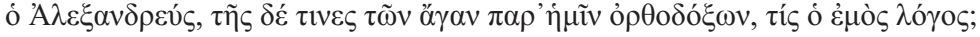

8. Cf. Ga 3, 28: «il n'y a pas de Grec ni de Juif... » et Col 3, 11 : «il n'y a pas de Grec et de Juif, de circoncision et de prépuce... ».

9. Grégoire de Nazianze, Discours, XXV, 16, éd. Justin Mossay, («Sources

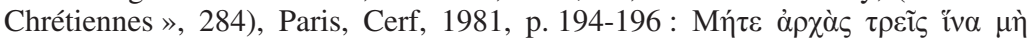

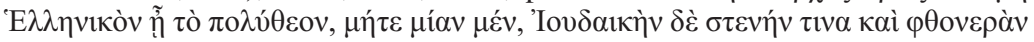


Grégoire place à l'intérieur de la catégorie du judaïsme : le sabellianisme (qui efface la notion d'hypostase et donc dissout le Fils dans le Père) et l'arianisme (qui abolit la divinité du Fils). La catégorie du polythéisme est celle du trithéisme, qui apparaît comme la déviance des ultra-orthodoxes. L'avantage de cette présentation saute aux yeux. En supprimant la catégorie de l'athéisme, elle fait apparaître que la voie royale, la voie médiane (non définie) se situe entre hellénisme/paganisme et judaïsme. C'était proprement la position d'Eusèbe de Césarée à laquelle semble se rallier ici Grégoire. Cette position ouvre le champ en rendant libre la catégorie de l'athéisme. Mais elle suppose toujours que le judaïsme correspond au monothéisme puisqu'il est question d'un «judaïsme étroit» comme si l'orthodoxie nicéenne était $a$ contrario un judaïsme large ou une richesse juive. En faisant du judaïsme la catégorie qui définit le monothéisme, la position de l'orthodoxie nicéenne apparaît toujours dépendante de son modèle juif, et suspendue dans le vide. Il n'y a toujours pas de pensée positive d'une position chrétienne dans un discours des religions.

On ajoutera que Grégoire accuse les Ariens de craindre une rébellion des natures contre la divinité. Autrement dit, on entend ici la critique arienne qui accuse la théologie nicéenne de «guerre civile » divine : en faisant du Fils et de l'Esprit, deux natures divines (on dirait plutôt aujourd' hui deux hypostases divines), on introduisait le risque de rébellion ou dissension (de stasis pour le dire en grec) à savoir que le Père, le Fils et l'Esprit "s'opposent entre eux » $(\grave{\alpha} v \tau \varepsilon \xi \hat{\alpha} \gamma \omega v \tau \alpha l)$. Toute la théorie politique grecque est sous-jacente à

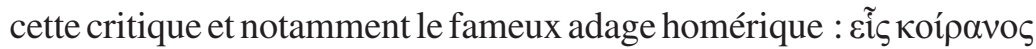
ह̌ $\sigma \tau \omega$, «qu'il n'y ait qu'un maître !». La réponse de Grégoire est une défense de la transcendance divine «plus puissante que les

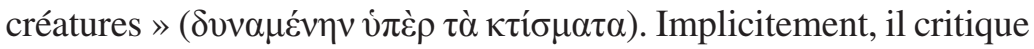
la réduction de la catégorie religieuse sous-jacente de monarchie à la catégorie politique de monarchie. C'est paradoxal, puisque Grégoire, lui, n'employait pas cette catégorie dans ce passage mais celle de judaïsme. Mais c'est bien à travers cette catégorie de monarchie et la peur panique de toute rébellion ou dissension que l'on peut

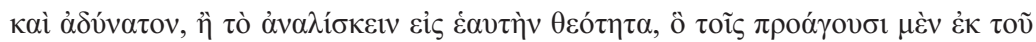

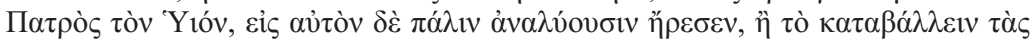

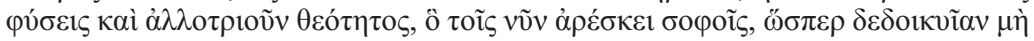

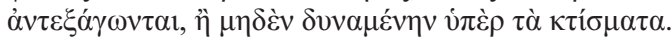


comprendre la critique du judaïsme. Il souffrirait «d'une certaine

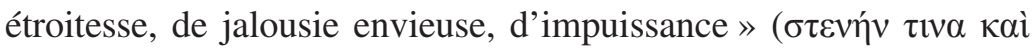

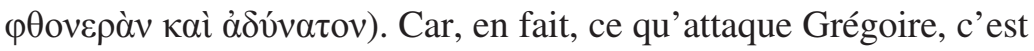
une maladie théologique, qui rend la monarchie divine similaire à la tyrannie laquelle est chez Aristote, Politique, IV, 2, 1 (1289a) une déviation de la monarchie. Le monarque divin est alors présenté comme un tyran qui ne veut s'entourer de personne par crainte d'un coup d'État et s'isole donc en lui-même dans une solitude haineuse. Refusant de recourir à un indispensable personnel politique, le tyran sombre dans l'impuissance.

L'erreur viendrait selon Grégoire justement de la conception humaine trop humaine de la divinité sur le modèle politique, alors que Dieu est au-delà de toute rébellion. La catégorie de monarchie est donc implicitement transcendée.

\subsection{Discours politico-religieux : monarchie, polyarchie, anarchie}

Le saut qualitatif est fait dans le Discours 29 de la même année 380. Comme la meilleure défense, c'est l'attaque, Grégoire change son fusil d'épaule et retourne complètement les idées de monarchie et de dissidence contre ses adversaires dans ce qui est peut-être le plus beau texte théologico-politique de l'histoire du christianisme :

Trois sont les plus anciennes opinions sur le dieu, anarchie, polyarchie et monarchie. De fait, les deux [premières] ont été le jeu des païens/hellènes : qu'ils continuent leur petit jeu! Ce qui est anarchique est privé d'ordre ; ce qui a plusieurs pouvoirs/principes appelle la guerre civile, et ainsi il devient anarchique et privé d'ordre : anarchie et polyarchie conduisent au désordre, et le désordre à la destruction, car le désordre est mise en pratique de la destruction. Pour nous, ce que nous honorons, c'est la monarchie : une monarchie certes, mais non celle qui se limite à une seule personne - car il arrive que même l'un entre en dissension avec lui-même ${ }^{10}$ et se fasse multitude $-{ }^{11}$.

10. Autre traduction : «car même l'un est dissident avec lui-même ».

11. Grégoire de Nazianze, Discours, XXIX, 2, éd. Pierre Gallay, («Sources

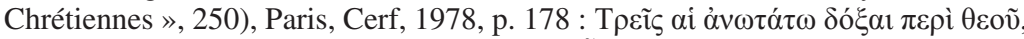

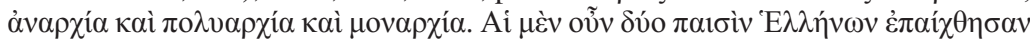

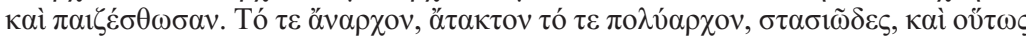

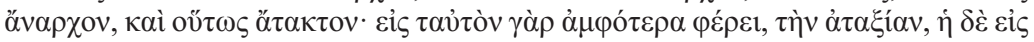

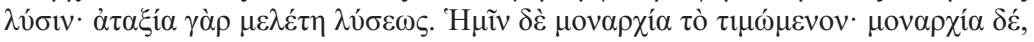

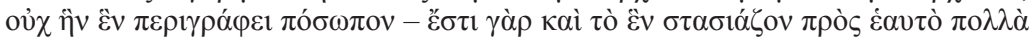
$\kappa \alpha \theta i ́ \sigma \tau \alpha \sigma \theta \alpha 1-$. 
La transformation s'est faite d'un discours sur les religions à un discours politique qui lui seul va permettre ce coup de force de mettre hors-jeu le judaïsme ${ }^{12}$. Le christianisme, en tant qu'il est l'orthodoxie nicéenne, peut alors devenir éventuellement la catégorie centrale entre le paganisme (ou plutôt en grec l'hellénisme), et le judaïsme. Coup double donc ! Cela n'était pas chose entièrement nouvelle pourtant, puisque Grégoire, fidèle à la tradition judéochrétienne depuis Philon jusqu'à Marcel d'Ancyre ${ }^{13}$, emploie les catégories politiques: anarchie, monarchie, polyarchie ${ }^{14}$, pour parler des opinions sur Dieu.

Mais le christianisme et l'orthodoxie nicéenne qui étaient absents des catégorisations précédentes peuvent enfin trouver leur place et le judaïsme qui apparaissait comme la «voie royale» est délogé. Tout cela n'a été rendu possible que par l'exclusion de l'athéisme hors du discours sur les religions. L'athéisme apparaît alors comme le zéro qui va permettre au système de se mettre en marche. Coup de maître ! En passant du discours des religions au discours politique, le polythéisme peut être défini comme polyarchie. Cette polyarchie, selon un principe politique traditionnel, peut-être réduite à l'anarchie. Coup de théâtre :

12. Bien que le mot ne soit pas employé, la mise en perspective avec les deux passages précédents conduit à bien voir que c'est le judaïsme prétendument « étroit » qui est visé à travers le concept de monarchie limitée à une personne, et derrière le judaïsme, l'arianisme.

13. Tout comme Philon d'Alexandrie, De confusione linguarum, 170, et Legatio ad Gaium, 149, le Pseudo-Justin, en fait Marcel d'Ancyre, au début du IV $^{\mathrm{e}}$ siècle, citait dans sa Cohortatio ad Graecos, XVII, (Pseudo-Justin, Ouvrages apologétiques, éd. Bernard Pouderon, («Sources Chrétiennes », 528), Paris, Cerf, 2009, p. 188) ce vers de l'Iliade, II, 204, très fameux dans l'Antiquité : «Il n'est pas bon qu'il y ait abondance de souverains, qu'un seul soit souverain » (ov̉к

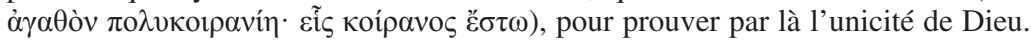
La tradition politique grecque attestée par cette citation est appelée en renfort du discours religieux juif ou chrétien, ce qui n'est pas le moindre des paradoxes. Sur l'usage de ces catégories chez Eusèbe de Césarée, voir Jean Sirinelli, Les vues historiques d'Eusèbe de Césarée durant la période pré-nicéenne, Paris, Université de Paris, 1961, p. 335.

14. Le mot a été introduit en anglais par Robert A. Dahl, A Preface to Democratic Theory, Chicago-Londres, University of Chicago Press, 1956. Le mot apparaît en français dans son sillage chez Alan Haye, «Les théories pluralistes de la démocratie dans la science politique américaine : une approche généalogique », Mémoire(s), identité( $s)$, marginalité(s) dans le monde occidental contemporain, [En ligne], 11 I 2014, Qui gouverne aux États-Unis ?, § 27 (mis en ligne le 15 mai 2014, consulté le 07 décembre 2015. URL : http://mimmoc. revues.org/1440). 
le polythéisme est donc réduit à l'athéisme, ce qui n'a pu être fait que par le recours aux catégories politiques. Mais nouveau coup de théâtre : si l'on interprète bien ce dernier texte avec les précédents, le judaïsme lui-même est réduit à un polythéisme par le concept central de stasis, de « dissidence », de « rébellion » ou même de «guerre civile». De même que le polythéisme est selon la tradition judéo-chrétienne qui le rattache à la tradition politique grecque de polyarchie, une dissidence, de même par un tour de force de Grégoire, le judaïsme est rattaché à une monarchie dissidente, et cela, contre la tradition politique grecque et le discours judéo-chrétien sur les religions.

Cette dernière phrase : "une monarchie certes, mais pas celle qui se limite à une seule personne »a, entre autres textes, permis à Erik Peterson de poser le dépassement par une théologie trinitaire de toute théologie politique ou de toute justification de la monarchie impériale dans sa fameuse étude Der Monotheismus als politisches Problem ${ }^{15}$. Mais Federico Fatti ${ }^{16}$ a récemment déconstruit l'argumentation de Peterson et montré que cette phrase, loin d'être un dépassement de la théologie politique, est au contraire une habile justification de la situation politique de l'époque. L'empereur Théodose qui établit en 380 Grégoire de Nazianze comme patriarche de Constantinople, règne alors en Orient, et Gratien en Occident : il s'agit donc d'une monarchie double, «qui ne se limite pas à une seule personne».

Tenant compte de ce contexte politique très particulier, revenons sur l'argumentation logique. Pour Grégoire, il s'agit de démontrer que seule l'orthodoxie chrétienne qui représente une forme de monarchie tempérée, permet d'éviter l'anarchie religieuse. La construction du raisonnement repose sur deux syllogismes sous-jacents :

15. Nous n'avons pas eu accès directement ni à l'ouvrage d'Erik Peterson, Der Monotheismus als politisches Problem, Ein Beitrag zur Geschichte der politischen Theologie im Imperium Romanum, Leipzig, Hegner, 1935 ni plus loin à celui de Carl Schmitt, Politische Theologie. Vier Kapitel zur Lehre von der Souveränität, Munich-Leipzig, Duncker \& Humblot, 1922, complété en 1934 et amplifié en 1969. Nous renvoyons à la brillante analyse de Federico Fatti, «Tra Peterson e Schmitt, Gregorio Nazianzeno e la "liquidazione di ogni teologia politica" ", Teologie politiche. Modelli a confronto, éd. Giovanni Filoramo, Brescia, Morcelliana, 2005, p. 61-101, ici p. 74-76.

16. F. Fatti, «Tra Peterson e Schmitt», p. 95. 
Premier syllogisme :

1 l'anarchie, c'est le désordre,

2 or la polyarchie mène à l'anarchie,

3 donc la polyarchie mène au désordre.

Deuxième syllogisme (double) :

4 il y a deux types de monarchie : stricte ou large,

5 et dans la monarchie stricte, le principe est l'un,

6 or l'un parfois entre en dissension avec lui-même et se fait multitude,

7 donc la monarchie stricte est une polyarchie,

8 or il a été démontré que la polyarchie mène au désordre,

9 donc la monarchie stricte mène au désordre.

Cette argumentation est implicite chez Grégoire, je l'explicite pour la clarté de l'exposé. Deuxièmement, la majeure du second syllogisme concernant l'existence de deux types de monarchie (proposition 4) semble pouvoir être rapportée à la Politique III, 16 (1287b) d'Aristote qui oppose une monarchie absolue à une

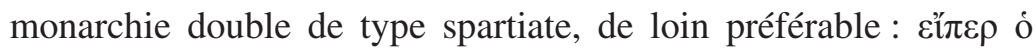

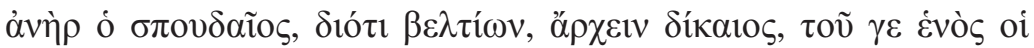

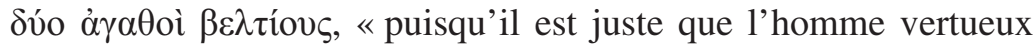
gouverne parce qu'il est le meilleur, deux hommes de bien sont meilleurs qu'un seul ».

Troisièmement, la mineure du second syllogisme (proposition 6) présente dans mon explicitation un défaut. On peut traduire la

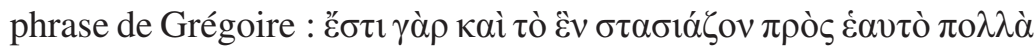
$\kappa \alpha \theta i ́ \sigma \tau \alpha \sigma \theta \alpha 1$, « car il arrive que même l'un entre en dissension avec lui-même et se fasse multitude ». Mais le raisonnement repose alors sur un risque et non sur un fait. On ne peut logiquement passer de cette proposition 6 à la proposition 7 selon laquelle la monarchie stricte est une polyarchie. Ou alors, il faut comme le fait Carl Schmitt dans sa réponse à Peterson, simplifier la phrase en $\tau o ̀$ ह̂v

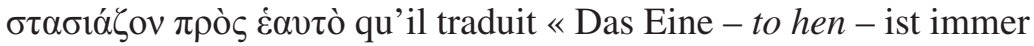
im Aufruhr - stasiatson - gegen sich selbst - pros heauton $-\gg^{17}$ : «l'un est toujours en révolte avec lui-même ». C'est forcer un peu

17. C. Schmitt, Politische Theologie, vol. II, p. 116. Voir F. Fatti, «Tra Peterson e Schmitt », p. 74. 
la syntaxe et cela lui a été reproché ${ }^{18}$; mais on peut faire aller $\tilde{\varepsilon} \sigma \tau 1$ avec $\sigma \tau \alpha \sigma i \alpha ́ \zeta o v$ et traduire « car l'un aussi est en dissension contre lui-même au point de se faire multitude ». Schmitt essaie ainsi de faire apparaitre « eine wahre politisch-theologische Stasiologie im Kern der Lehre von der Trinität $»^{19}$. C'est tout à fait vrai puisque quelques lignes plus loin, Grégoire résume en une sentence bien martelée ce mouvement, on dirait volontiers cette stasis de l'un

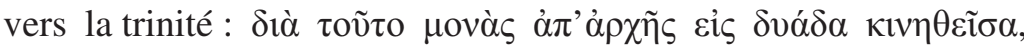
$\mu \varepsilon ́ \chi \rho 1 \tau \rho t \alpha ́ \delta o \varsigma$ है $\sigma \tau \eta$, «pour cela, l'unité dès l'origine s'est mue en dualité et s'est arrêtée en trinité ». De fait, cette idée paradoxale de la stasis ou du mouvement de l'un repose peut-être sur l'expression du Parménide de Platon ${ }^{20}$. Parménide montre que l'un se divise en deux (l'un et l'être), dans la mesure où l'un ne participe pas au temps qui est une condition de l'être :

L'un possède toujours l'être, et l'être toujours l'un, de sorte que nécessairement, comme il devient toujours deux, il n'est jamais un. - C'est absolument sûr. - Il s'ensuit que puisque la multitude est infinie, ainsi l'un, étant la multitude, serait infini ? - C'est à croire ${ }^{21}$.

Dans ce cas, et ce n'est pas le moindre paradoxe, c'est en recourant implicitement ${ }^{22}$ à la tradition philosophique platonicienne et néo-platonicienne que Grégoire développe son raisonnement. En effet, le texte de Grégoire pourrait être une réinterprétation ${ }^{23}$ d'un texte des Ennéades $(\mathrm{V}, 2,1)$ de Plotin qui semble lui-même se

18. Voir les références chez F. Fatti, «Tra Peterson e Schmitt», p. 75, n. 47.

19. C. Schmitt, Politische Theologie, vol. II, p. 118. Cela signifierait pour Schmitt que la notion d'ami/ennemi qui est au centre de sa pensée politique serait bien dans la conception grégorienne de la Trinité, et qu'elle serait donc irrémédiablement politique. Voir F. Fatti, «Tra Peterson e Schmitt», p. 74-75.

20. C. Schmitt, lui, renvoie pour la notion de stasis à la République, 470 (où elle a le sens de dissidence) et au Sophiste, 249-254 (où elle a le sens de repos). Voir F. Fatti, « Tra Peterson e Schmitt », p. 74.

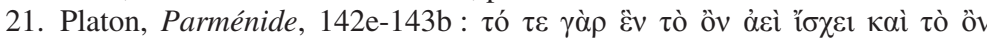

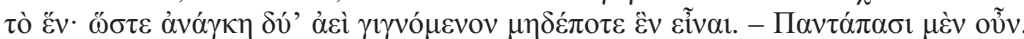

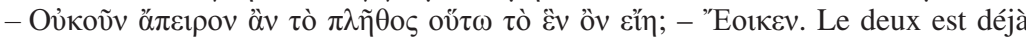
multitude...

22. Quelques lignes plus loin en effet (Discours, XXIX, 2), Grégoire se défend explicitement de définir la procession divine de la première cause à la seconde cause sur le modèle du débordement ( $\dot{\varepsilon} \xi \varepsilon \rho \rho v ́ \eta)$ d'un cratère, ce qui fait allusion aux Ennéades de Plotin, V, 1, 6.

23. Nous devons cette allusion au commentaire de Joshua Lollar, Maximus the Confessor, Ambigua to Thomas, Second letter to Thomas, Turnhout, Brepols, 2009, p. 95, n. 8, qui se réfère à Frederick W. Norris, Faith Gives Fullness to Reasoning, Leiden, Brill, 1991, p. 134-135. 
référer au Parménide : «Et sa dissidence vis-à-vis de l'un a produit l'étant, et la vision [de l'étant a produit] l'intelligence » (Kaì ì $\mu \bar{v} v$

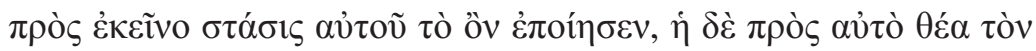
voṽv $)^{24}$. Ici, Grégoire fait subir au monothéisme juif la critique du Parménide. Si du point de vue de la philosophie des religions ou de la rhétorique politique, les affirmations de Grégoire de Nazianze sont passionnantes, d'un pur point de vue logique, elles sont très problématiques.

Revenons au texte.

1. Grégoire dit : « Ce qui est anarchique est privé d'ordre ; ce qui a plusieurs pouvoirs/principes appelle la guerre civile $(\sigma \tau \alpha \sigma i \tilde{\omega} \delta \varepsilon \varsigma)$, et ainsi il devient anarchique et privé d'ordre».

2. Il continue un peu plus loin : « car même l'un est en dissidence

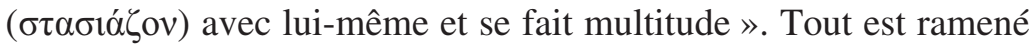
à l'anarchie par le biais du concept finement mis en valeur par Schmitt de stasis. Le polythéisme appelle la stasis, la «guerre civile» des dieux et il devient anarchie divine, mais le monisme judaïque lui-même se fait stasis, « guerre avec lui-même » et tombe dans l'anarchie. Pourquoi pas ? Rien à redire jusque-là...

3. Mais Grégoire continue en disant quelques phrases plus loin que «pour cela, l'unité dès l'origine s'est mue en dualité et s'est arrêtée en trinité ». Or si l'on rapporte cette affirmation à la précédente, on aboutit à un problème insurmontable. En effet, c'est la dissidence devenue mouvement de l'un qui a conduit à la trinité. Mais la dissidence de l'un est aussi ce qui implicitement conduit au multiple et donc au polythéisme en guerre civile contre

24. Y a-t-il (ré)interprétation de $\sigma \tau \alpha ́ \sigma 1 \varsigma$ par Grégoire comme «dissidence », du fait de la préposition $\pi \rho$ ò $\varsigma$ suivie d'un accusatif de mouvement? Maria Isabel Santa Cruz de Prunes, La genèse du monde sensible dans la philosophie de Plotin,

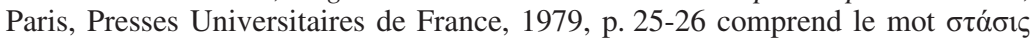
au sens de «repos » et explique : «De l'Un surgit un produit qui est à la fois être et intelligence. Ce produit est l'être dans la mesure où il reste en repos près de l'Un et il est intelligence dans la mesure où il se tourne vers son principe et le contemple activement». Cependant, la traduction d'Arthur H. Armstrong, Plotinus, Enneads, vol. v, Cambridge, Mass.-Londres, Harvard University Press, 1984 , p. 59 : «Its halt and turning towards the One constitutes being, its gaze upon the One, Intellect » montre combien le sens de « repos » n'est pas suffisant, pour le moins, puisqu'il ajoute la notion de « retour », ce qui paraît contradictoire avec la notion de « repos ». Jean-Marc Narbonne, Plotin, les deux matières [Ennéades II, 4, 12], Paris, Vrin, 1993, p. 139, en tout cas insiste sur le fait qu' « à l'inverse de son condisciple Origène [le platonicien] resté fidèle apparemment sur ce point à Ammonius, Plotin [a] séparé son premier principe, l'Un, de l'Intelligence... ». 
lui-même. En d'autres termes, le christianisme apparaît comme une étape de l'audacieuse réduction du judaïsme au polythéisme, et par là à l'anarchie qui tient lieu d'athéisme. Et donc, implicitement certes, mais en bonne logique, le christianisme est entraîné avec le judaïsme vers le polythéisme...

\section{Le discours Sur les Religions de Maxime le Confesseur}

\subsection{Le refus de la stasiologie par Maxime le Confesseur}

Comme l'a rappelé Caroline Macé25, Grégoire de Nazianze est devenu à partir du $\mathrm{VI}^{\mathrm{e}}$ siècle l'autorité suprême en matière de

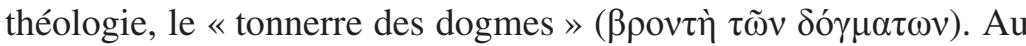
$\mathrm{VII}^{\mathrm{e}}$ siècle, Maxime le commente à l'égal de la Bible. Il a expliqué quatre fois ${ }^{26} 1$ 'énigmatique phrase de Grégoire : «pour cela, l'unité dès l'origine s'est mue en dualité et s'est arrêtée en trinité ».

Dans le premier Ambiguum ad Thomam qui remonte à l'année 634 ou 635, il affirme :

la divinité est Trinité et non pluralité, en tant qu'elle est sans commencement, sans corps et sans dissidence intérieure ${ }^{27}$.

Il récuse ainsi l'idée de pluralité pour parler de la Trinité et celle de dissidence pour définir la divinité. Maxime vide le texte de Grégoire : la notion de stasis évoquée par Grégoire quelques lignes avant ce passage est tout simplement évacuée en tant que la divinité ne peut être que dépourvue de toute dissidence interne

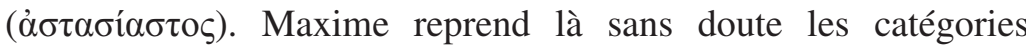

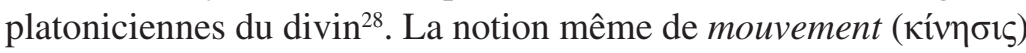

25. Caroline Macé, "Gregory of Nazianzus as the authoritative voice of Orthodoxy in the sixth Century », Byzantine orthodoxies, éd. Andrew Louth, Augustine Cassiday, Aldershot, Ashgate, 2006, p. 33-34.

26. Dans le premier Ambiguum ad Thomam, dans le premier point de la Deuxième lettre à Thomas, dans l'Ambiguum ad Johannem, 23 et dans les Quaestiones et dubia, 105.

27. Maximi Confessoris, Ambigua ad Thomam, éd. Bart Janssens, («Corpus Christianorum Series Graeca», 48), Turnhout-Louvain, Brepols, 2002, p. 7,

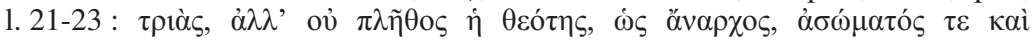
$\dot{\alpha} \sigma \tau \alpha \sigma i \alpha \sigma \tau \sigma \zeta$.

28. À la fin du deuxième livre de la République (380c-383c), Platon fait poser à Socrate trois lois concernant le dieu : il n'est cause que des biens, il est impossible même pour un dieu de s'altérer lui-même, il est absolument étranger au mensonge. 
qui ne fait que développer l'idée de stasis est récusée au profit d'une conception immobile et immuable de la divinité, dont le mouvement n'est qu'une apparence de mouvement. Ce serait en fait le mouvement de l'esprit humain qui tente de comprendre la divinité mais non pas un mouvement en Dieu.

C'est là revenir à la position première et inconfortable de Grégoire où le judaïsme restait un obstacle à l'affirmation du monothéisme chrétien. Malgré sa prétendue «étroitesse », le judaïsme occupe toujours le terrain.

\subsection{Le discours binaire sur les religions de Maxime : hellénisme-polythéisme et judaïsme-athéisme}

Dans un autre texte, la Brève interprétation du Notre Père, écrite avant 636, Maxime propose une autre solution. Il reprend le discours binaire de Grégoire (hellénisme et judaïsme). C'est en fait le discours paulinien sur les religions (devenu traditionnel dans le christianisme) qui suppose la transcendance du christianisme par rapport aux autres religions ${ }^{29}$.

Le coup de maitre de Maxime consiste à identifier, en bon polémiste, le judaïsme directement à l'athéisme. Cette catégorie absente du discours binaire de Grégoire sur les religions permettait à son système de fonctionner. Il autorisait une réduction de la polyarchie polythéiste à l'anarchie et aussi de la monarchie stricte juive à la polyarchie polythéiste et à l'anarchie. Maxime s'est toujours refusé à la solution de Grégoire qui lui paraissait trop coûteuse en termes théologiques puisqu'elle associait dangereusement la Trinité à la pluralité et la divinité à la dissidence, deux reproches justement adressés au polythéisme hellène/païen. Ce que propose Maxime est un court-circuit. La monarchie juive est réduite directement à l'anarchie, ou plutôt en termes maximiens à l'athéisme. Voici le texte :

«Ni Grec ni Juif » (Ga 3, 28; Col 3, 11), à travers lesquels sont désignés les discours variés, ou pour le dire avec plus de vérité, contraires de l'opinion qu'on a de Dieu. Le premier introduit de façon

29. Maxime cite en effet Paul dans l'épître aux Galates $(3,28)$ : «Il n'y a pas de Grec ni de Juif, il n'y a pas d'esclave ni d'homme libre, il n'y a pas d'homme et de femme : car tous vous êtes un dans le Christ Jésus », et dans l'épître aux Colossiens $(3,11)$ : « là où il n'y a pas de Grec et de Juif, de circoncision et de prépuce, de barbare, de Scythe, d'esclave, d'homme libre, mais totalement et en tout le Christ ». 
insensée une polyarchie, divise le principe en activités et puissances contraires, forge un objet de vénération polythéiste qui se scinde en factions rivales du fait de la pluralité des êtres adorés et tombe dans le ridicule du fait des différents modes d'adoration. Le second introduit certes un unique [principe], mais qui est étroit, imparfait et presque sans subsistance, privé qu'il est de raison et de vie, et ce discours tombe dans un malheur égal au précédent discours, mais pour des raisons opposées, à savoir l'athéisme. Il limite à une seule personne le principe unique et [le fait] subsister sans raison ni esprit ${ }^{30}$.

Maxime combine en fait le discours binaire de Grégoire de Nazianze sur les religions avec son usage novateur des catégories théologico-politiques ${ }^{31}$. La nouveauté est que Maxime veut, lui, enfermer le judaïsme dans l'athéisme. Cela lui permettrait implicitement de présenter le christianisme comme la voie moyenne entre polythéisme païen et un athéisme juif, à un moment où, au $\mathrm{VII}^{\mathrm{e}}$ siècle, la pratique païenne est en voie de disparition tandis le judaïsme connaît un regain de vigueur et devient un concurrent réel du christianisme ${ }^{32}$.

L'argumentation que donne Maxime pour justifier sa position repose sur les progrès de la logique dans le discours théologique

30. Maximi Confessoris, Opuscula exegetica duo, éd. Peter Van Deun, ( Corpus Christianorum Series Graeca », 23), Turnhout-Louvain, Brepols, 1991,

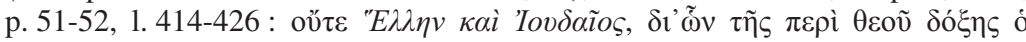

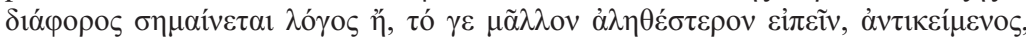

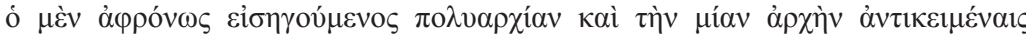

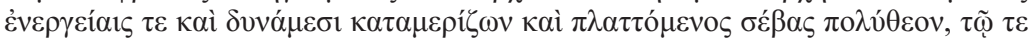

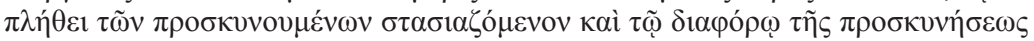

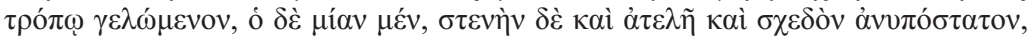

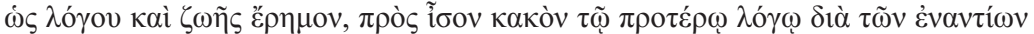

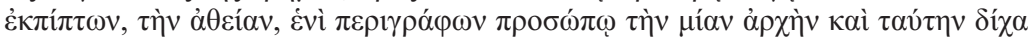

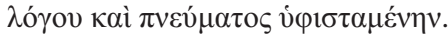

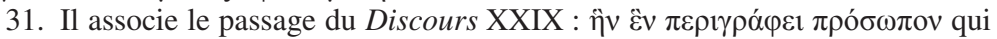
devient chez lui : $\dot{\varepsilon} v \grave{i} \pi \varepsilon \rho \imath \gamma \rho \alpha ́ \varphi \omega v \pi \rho o \sigma \omega ́ \pi \omega$, en la combinant avec une citation du

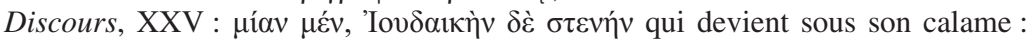

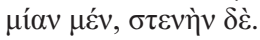

32. Voir Carl Laga, «Le judaïsme et les juifs dans l'œuvre de Maxime le Confesseur », Byzantinoslavica, 51, 1990, p. 177-188. On note une effervescence apocalyptique chez les Juifs vers 630-640: Apocalypse de Zorobabel, Les signes du Messie, les poèmes de Qalir/Qilir, cf. Gilbert Dagron, Vincent Déroche, Juifs et chrétiens en Orient byzantin, Paris, Association des Amis du Centre d'Histoire et Civilisation de Byzance, 2010, p. 41. C'est au VII siècle que Peter Schäfer, Die Geburt des Judentums aus dem Geist des Christentums, Tübingen, Mohr Siebeck, 2010, p. 2, place la rédaction du Talmud de Babylone (Talmud Bavli). Ces productions littéraires sont le signe d'une nouvelle affirmation de soi des Juifs au $\mathrm{VII}^{\mathrm{e}}$ siècle et d'un renouveau des polémiques judéo-chrétiennes. 
à travers la notion d'enhypostaton ${ }^{33}$. Aux substances premières d'Aristote, individuelles (par exemple Socrate), Maxime substitue les hypostases; seules les substances secondes d'Aristote, générales (par exemple l'homme) sont pour lui des substances. Les substances, tout comme les qualités et les accidents, n'existent que dans et par les hypostases, comme substances insubsistantes

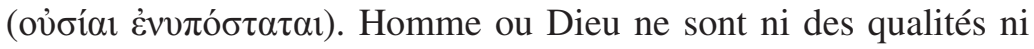
des accidents qui définissent les hypostases, mais des substances insubsistantes: elles existent dans (in-subsistantes) et par les hypostases que sont par exemple Pierre et Paul, ou le Père, le Fils et l'Esprit. À l'inverse, une substance qui n'a pas de représentant existant, c'est-à-dire d'hypostase, est une substance non-subsistante

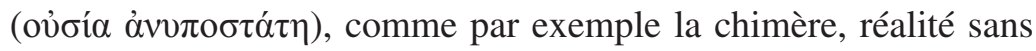
existence individuelle.

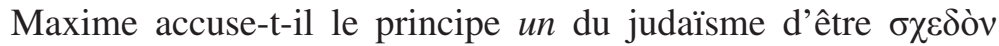
$\alpha \grave{\alpha v} \pi$ ó $\tau \alpha \tau o v$, « quasiment anhypostaton » ou «presque dépourvu de subsistance » pour la simple raison qu'il lui reprocherait de priver Dieu d'existence et d'en faire une abstraction, au point de sombrer dans l'athéisme? Non, car on pourrait imaginer un singleton : la substance Dieu, substance insubsistante dans une unique existence qui serait le Père. De fait, c'est ce qui arrive puisque le principe un est «limité à une seule personne », c'est-à-dire à une seule hypostase ou existence.

Alors, où est le problème ? Le principe un, le premier principe juif serait $\dot{\alpha} \tau \varepsilon \lambda \eta ́ s$, «imparfait», dit Maxime. Ne serait-on pas là en fait encore dans la tradition du Parménide de Platon qui pose la question de l'être de l'un ? Le premier principe, l'un, le Dieu juif, ne serait-il pas privé de l'être ? Le risque de dualité de l'un avait conduit Plotin (Ennéades, V, 5, 6) contre son maître Ammonius à poser l'existence d'un premier un, transcendant, séparé de l'être, c'est-à-dire d'un second un, l'un-être. Et ce premier un en tant qu'il est transcendant ne recevait aucun qualificatif, aucun nom

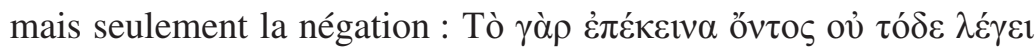

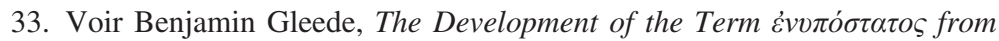
Origen to John of Damascus, Leiden, Brill, 2012 : nous lui empruntons le mot

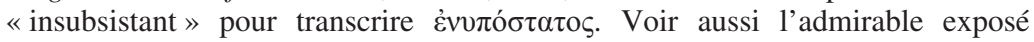
synthétique de Christophe Erisman, «A world of hypostasis, John of Damascus' Rethinking of Aristotle's Categorical Ontology », Studia patristica, 50, 2011, p. 269-287. 


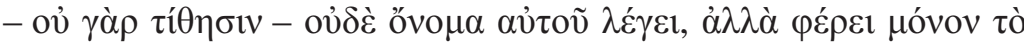

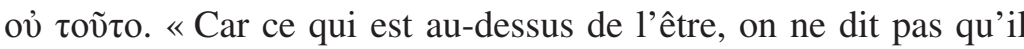
est ainsi (car il ne l'assume pas) et il ne dit pas son nom : mais il supporte seulement qu'on le décrive comme : pas ainsi $»$.

Cette position transcendantale n'a, semble-t-il, pas convenu à Porphyre dans son commentaire au Parménide « qui paraît presque hérétique par rapport à la tradition du néoplatonisme issu de Plotin $»^{34}$. Au lieu de séparer ce premier un de tout le reste comme le font et son maître Plotin et les autres néo-platoniciens, Porphyre pose que le premier un est l'être, l'acte d'être, tandis que le second un est l'étant qui participe à l'être du premier un. Ainsi au lieu d'être absolu et transcendant, le premier un était participé par le second un, l'un-qui-est. Et même Porphyre faisait de ce premier $u n$ et de ce second un deux états de l'intelligence. Alors que Plotin posait le premier un, l'un transcendant, et ensuite, sans participation à lui, faisait apparaitre l'être qui se sépare de l'un, et l'intelligence, Porphyre, lui, proposait une autre solution. Le premier un était identifié à l'intelligence pure (premier état de l'intelligence). Mais quand cette intelligence entrait en existence (deuxième état de l'intelligence), elle se mettait à penser sa pensée, et voulant atteindre ce qu'elle pense, elle devenait vie ; ainsi naissait la triade : existence, pensée, vie. Porphyre renonçait donc à la séparation transcendantale entre le premier un et le reste. Dans ce système, l'entrée de l'un dans l'existence conduisait à une trinité : existence, pensée, vie, celle-là même que suppose Maxime quand il parle, en plus du premier principe, de raison et de vie.

Si l'on accepte que Maxime critique le monothéisme juif, en refusant la solution transcendantale plotinienne d'un Dieu qui serait sans rapport avec aucune existence pour préférer un système porphyrien, alors la position maximienne est peut-être discutable mais prend sens. Reste cependant la question de la connaissance que Maxime a pu avoir de ce système : elle a pu être aussi bien directe qu'indirecte ${ }^{35}$.

34. Pierre Hadot, Porphyre et Victorinus, vol. I, Paris, Études Augustiniennes, 1968, p. 113.

35. La doctrine de la Trinité chrétienne s'est volontiers coulée dans ce système porphyrien, où Dieu, bien que transcendant, n'est pas séparé de ses trois hypostases. Déjà Grégoire de Nysse, comme le rappelle S. Lilla, Dionigi l'Areopagita, p. 117, n'hésite pas contre Plotin à attribuer l'être à Dieu selon une doctrine qui remonte à 
En tout cas, et c'est là le paradoxe, c'est en reprenant les spéculations de la tradition platonicienne (et singulièrement de Porphyre) que Maxime accuse le judaïsme d'être un athéisme. Je ne suis pas sûr que la pensée de Maxime soit parfaitement recevable d'un point de vue logique. Mais le résultat implicite est de poser le christianisme comme la voie royale entre l'athéisme juif et le polythéisme hellène / païen. On a là un tour de force, qui voudrait achever le triomphe religieux du christianisme en effaçant le judaïsme du monothéisme. Le christianisme pourrait alors apparaître comme le seul vrai monothéisme, en ce viI ${ }^{\mathrm{e}}$ siècle, moment de lutte intense entre les deux monothéismes juif et chrétien, qui donnera naissance à un troisième monothéisme, musulman.

En conclusion, on rappellera que le mot de « monothéisme », qui n'existe pas encore, se dit en grec $\mu$ ov $\alpha \rho \chi i ́ \alpha$, et que l'on ne peut pas penser la catégorie religieuse de monarchie qui correspond grosso modo à celle de notre monothéisme sans penser en même temps la question politique de la monarchie ${ }^{36}$. Monothéisme peut aussi se dire en grec, non sans ambiguïté, par judaïsme, dans un discours traditionnel des religions binaire : judaïsme, hellénisme ; voire, dans un discours ternaire : judaïsme, hellénisme-polythéisme, athéisme. $\mathrm{Ce}$ dernier type de discours recoupe la forme politico-religieuse du discours sur les religions: monarchie, polyarchie, anarchie. Le christianisme n'est pas présent à l'intérieur de ce discours des religions comme une catégorie particulière, mais apparaît comme un dépassement transcendant des « religions » (on devrait plutôt dire théologies), en tant qu'opinions sur Dieu.

La cible de Grégoire de Nazianze n'est pas le judaïsme, mais les hérésies. Le christianisme nicéen revendique alors une position transcendante par rapport aux hérésies et leur modèle tiré du discours

Numénius, Porphyre et Origène le platonicien. Il pense aussi la génération du Fils sur le modèle dont l'intelligence produit la parole...

36. Cela n'est pas sans évoquer notre époque démocratique et sa critique de la monarchie divine, ou plutôt du monothéisme, comme fanatisme totalitaire. Voir déjà l'association polémique du bolchévisme et du mahométanisme dans le livre de Bertrand Russell, The Practice and Theory of Bolshevism, Londres, Allen and Unwin, 1920, p. 114 : " Mahommedanism and Bolshevism are practical, social, unspiritual, concerned to win empire on this world. Their founders would not have resisted the third of temptations in the wilderness ». Cette dernière phrase est probablement un trait d'humour britannique. 
sur les religions. Dans ce contexte hérésiologique, il y a deux critiques du monothéisme chez Grégoire, qui sont en fait des critiques de l'arianisme identifié au judaïsme. La première et principale critique faite par Grégoire au judaïsme est son «étroitesse ». Derrière cette catégorie de judaïsme, se trouve en réalité la catégorie de monarchie et sa critique en tant que tyrannie. La seconde critique par Grégoire du monothéisme repose peut-être sur l'idée de dissension que produit la monarchie étroite, la tyrannie. Reprenant la tradition philosophique du Parménide de Platon et réinterprétant sans doute la philosophie de Plotin, Grégoire envisage alors une dissension de l'un avec lui-même qui conduit à une pluralité de principes en dissension avec eux-mêmes jusqu'à leur anéantissement. Le christianisme trinitaire représenterait, position acrobatique, le seul moment stable dans ce processus de transformation de l'un en multiple et en néant. Cette critique philosophiquement géniale et historiquement défendable en 380 après J.-C. est en même temps rhétoriquement et théologiquement extrêmement paradoxale: le christianisme trinitaire reposerait sur une philosophie de la rébellion ${ }^{37}$, une stasiologie comme l'a compris Carl Schmitt.

À la suite de Grégoire et contre lui, Maxime récuse cette stasiologie comme contradictoire avec la notion de divinité, nécessairement dépourvue de toute dissension. Il élabore à partir de la première et de la seconde critique du monothéisme un nouveau discours sur les religions où le christianisme occupe désormais implicitement une position centrale entre le polythéisme païen et le judaïsme réduit à un athéisme. Il s'appuie sur le développement de la théologie néo-chacédonienne et en particulier sur la notion d'enhypostaton. Une réalité, une substance existe seulement dans la mesure où elle s'incarne en individus existants. Peut-être même Maxime reprend-il, directement ou indirectement, la philosophie de Porphyre, commentant le Parménide, qui concevait non pas l'un transcendant et séparé des êtres à la façon de Plotin, mais l'un qui entrait dans l'être en s'individualisant en existence, pensée et vie. En jouant Porphyre contre Plotin, Maxime accusait sans doute le Dieu du judaïsme d'être tellement transcendant qu'il n'entrait plus

37. Le christianisme serait à la fois un monothéisme anarchique (dans la mesure où l'un entre en dissension avec lui-même) et une réponse à l'anarchie monothéiste (dans la mesure où le trois poserait une fin à la dissension). 
dans le processus de différentiation existentielle, et donc d'être inexistant.

Est-ce que le platonicien de Cambridge que fut Henry More connaissait ces positions de Maxime ou de Grégoire quand il inventa le mot polémique de "monothéisme » dans son traité $A n$ explanation of The grand Mystery of Godliness en 1660 ? Sans doute au moins connaissait-il celles de Grégoire... Toujours est-il que, même s'il vise le panthéisme de la fin de l'Antiquité, hermétique ou néo-platonicien, il associe justement dans une même phrase le monothéisme à l'athéisme. "This Kinde of Monotheisme of the Heathen is as rank Atheisme as their Polytheisme was proved before $»^{38}$. N'a-t-on pas la même habile logique de réduction des religions à l'athéisme à l'œuvre chez Grégoire ou Maxime? L'origine du discours moderne sur les religions et du concept polémique de monothéisme serait-elle donc à chercher dans nos deux audacieux penseurs antiques ? Ce serait une piste intéressante à suivre, que nous réservons à d'autres... ${ }^{39}$

christian.boudignon@gmail.com

38. Henry More, An explanation of The grand Mystery of Godliness, Londres, J. Flesher, 1660, p. 62.

39. Nathan Mac Donald, "The Origin of "Monotheism" », Early Jewish and Christian Monotheism, éd. Loren T. Stuckenbruck, Wendy E. S. North, LondresNew-York, T\&T Clark International, 2004, p. 204-215 trace bien l'origine historique du concept au XVII ${ }^{\mathrm{e}}$ siècle mais ne souligne pas ce qui pourrait en être le background patristique. 